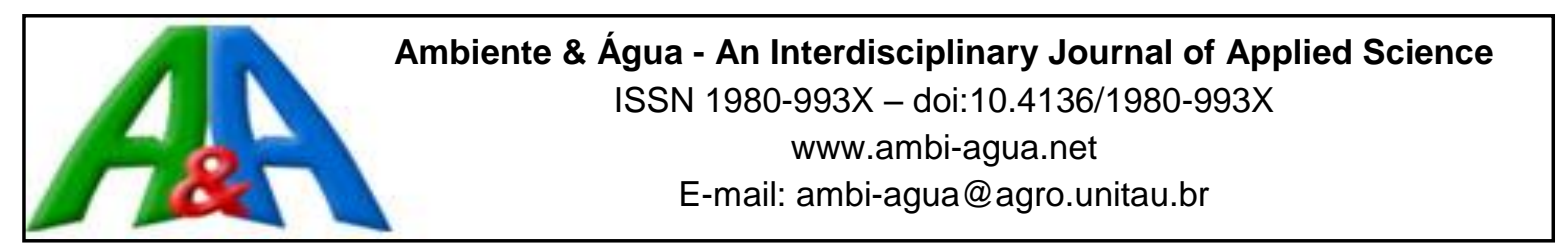

\title{
Calidad de las aguas meteóricas en la ciudad de Itajubá, Minas Gerais, Brasil
}

\author{
doi: 10.4136/ambi-agua.1329
}

Received: 26 Feb. 2014; Accepted: 20 May 2014

\author{
Herlane Costa Calheiros ${ }^{1}$; Mônica Regina Gomes ${ }^{1}$; \\ Percy Máximo Anco Estrella ${ }^{2}$ \\ ${ }^{\mathbf{1}}$ Universidade Federal de Itajubá (UNIFEI), Itajubá, MG, Brasil \\ Instituto de Recursos Naturais (IRN) \\ ${ }^{2}$ Universidad Nacional de Ingeníeria (UNI), Lima, Peru \\ Empresa PROWIL \\ *Autor correspondiente: e-mail: profaherlane@yahoo.com.br, \\ monicargomes@gmail.com, percy.anco@yahoo.com.br
}

\section{RESUMEN}

El objetivo de este estudio fue evaluar la calidad del agua meteórica en la ciudad de Itajubá, ubicado en la parte sur de Minas Gerais, Brasil. Para ello, se realizaron la colecta y análisis de la calidad de las muestras de agua de lluvia después de escurrir sobre la superficie de tres tipos de materiales; amianto, zinc y cerámica. También se realizó una colecta directa para comparar los resultados y verificar los cambios en la calidad del agua. Se observó que la calidad del agua de lluvia varía en función del material a través en el cual fluye, alterando de esta manera los parámetros físicos-microbiológicos como el $\mathrm{pH}$, dureza, alcalinidad, turbidez y el contenido de coliformes. Por lo tanto, el material de la superficie de los sistemas de colección de agua de lluvia puede modificar la calidad del agua, y el destino final de este tipo de agua. A pesar que las aguas meteóricas de Itajubá presentaron un bajo grado de contaminación, se concluye que el tratamiento de estas aguas deben prever la remoción de sólidos, el ajuste de $\mathrm{pH}$ y la desinfección, de esta forma, se estará obteniendo una alternativa ventajosa, sustentable y asequible de uso en época de escases hídrica.

Palabras-clave: disponibilidad de agua, agua de lluvia, aprovechamiento de agua pluvial, lluvia ácida, contaminación del aire.

\section{Qualidade das águas meteóricas no município de Itajubá, Minas Gerais, Brasil}

\section{RESUMO}

Este trabalho teve como objetivo avaliar a qualidade das águas meteóricas no município de Itajubá, localizado no Sul do Estado de Minas Gerais, no Brasil. Para tanto, realizou-se a coleta e análise da qualidade das águas de chuva após escoar por três materiais: amianto, zinco e cerâmica. A coleta direta também foi realizada para comparação dos resultados e verificação das alterações ocorridas na qualidade da água. Observou-se que a qualidade da água de chuva varia dependendo do material pela qual escorre, alterando parâmetros como $\mathrm{pH}$, dureza, alcalinidade, turbidez e concentração de coliformes. Portanto, o material da 
superfície para sistemas de coleta de águas pluviais pode modificar a qualidade da água e, portanto, o destino final desta água. Apesar das águas meteóricas de Itajubá apresentarem baixo grau de contaminação, conclui-se que o tratamento destas águas devem prever a remoção de sólidos, o ajuste do $\mathrm{pH}$ e a desinfecção, tornando-as uma alternativa vantajosa, sustentável e acessível de uso em época de escassez hídrica.

Palavras-chave: disponibilidade hídrica, água de chuva, aproveitamento de água pluvial, chuva ácida, poluição do ar.

\section{Quality of meteoric water in the city of Itajubá, Minas Gerais, Brazil}

\section{ABSTRACT}

The objective of this study was to assess the quality of rainwater in the city of Itajubá, MG. Therefore, samples were collected in order to analyze rainwater quality after the water had drained through three materials: asbestos, zinc and ceramic. Direct sampling was also performed in order to compare results and to verify changes in rainwater quality. It was observed that the quality of rainwater varies with the material through which it flows, changing parameters such as $\mathrm{pH}$, hardness, alkalinity, turbidity and coliform concentration. Therefore, the surface material in rainwater collection systems can modify water quality and thus affect the final suitable uses of this water. Although the meteoric waters of Itajubá had a low degree of contamination, it was concluded that the treatment of these waters must include the removal of solids, $\mathrm{pH}$ adjustment and disinfection, which will render them an advantageous, sustainable and affordable alternative for use in times of shortage water.

Keywords: water availability, rainwater, pluvial water harvesting, acid rain, air pollution.

\section{INTRODUCCION}

Las aguas meteóricas son aquellas provenientes del vapor de agua atmosférico que pueden precipitar en forma de neblina, lluvia, helada, nieve, granizo y entre otras formas.

El uso de los sistemas de aprovechamiento de las aguas meteóricas, según May (2004), es viabilizado para reducir el consumo de agua potable, disminuir los costos de agua suministrada por las empresas de abastecimiento y para minimizar los riesgos de inundación en caso de lluvias intensas, como método de preservación del medio ambiente, reduciendo la escases de recursos hídricos en épocas de estiaje. Al menos, algunos de estos beneficios han sido experimentados por varios países como Indonesia, Taiwan, Australia, Inglaterra, Tailandia, Quenia, Botswana, Japón, Perú, Alemania, Singapur, China, entre otros (Lee y Nazarudeen, 1996; Schimidt, 2001; Gnadling, 2001) y de la misma forma en diversas ciudades brasileiras, como por ejemplo; Ribeirão Preto, Rio de Janeiro, Salvador, Fernando de Noronha, Florianópolis, Joinville, desiertos y áreas secas de Pernambuco (Campos et al. 2003; Azevedo Netto, 1991; Ramos, 1983; Liberal y Porto, 1999) por tener experiencias con el aprovechamiento de las aguas meteóricas.

La composición de las aguas de lluvia varían de acuerdo a las regiones y, según Eaton et al. (1973), está relacionada con los lugares de origen de las masas de aire y la génesis de la lluvia. Thomas y Greene (1993), investigando la calidad de las aguas de lluvia en contacto con los diversos materiales de los techos en áreas rurales, urbanas e industriales, concluyeron que los materiales de los techos tienen influencia sobre la calidad del agua de lluvia y que las aguas pluviales contaminadas colectadas de los techos se debe principalmente a la contaminación difusa de la deposición atmosférica y del número de días secos que anteceden a un evento lluvioso. Kus et al. (2013) también verificaron que los factores como la intensidad 
del tráfico de vehículos, la contaminación del aire y la localización urbana o rural influyen en la calidad de las aguas pluviales.

Las aguas meteóricas con pH inferior a 5.6 son consideradas ácidas, según Jaques (2005), indicando que el agua se encuentra contaminada con ácidos fuertes así como el ácido sulfúrico $\left(\mathrm{H}_{2} \mathrm{SO}_{4}\right)$ y el ácido nítrico $\left(\mathrm{HNO}_{3}\right)$, y eventualmente con otros tipos de ácidos como el ácido clorhídrico $(\mathrm{HCl})$ y los ácidos orgánicos, siendo un problema por la acción corrosiva para materiales metálicos, calcarios y otras sustancias.

Los parámetros controlados en la calidad del agua para consumo dependen del uso al cual es destinado. El hombre utiliza agua para diversas finalidades, que van desde el propio consumo humano hasta como materia prima para la fabricación de diversos productos. En lo que se refiere al consumo en las residencias, el agua puede ser utilizada tanto para fines potables (higiene personal, preparación de alimentos y otros) y no potables (lavado de carros, riego de jardines, uso sanitarios, etc). Debido a sus características naturales, generalmente, presentan concentraciones bajas en minerales. Tomaz (2003) recomienda que las aguas meteóricas sean usadas para fines no potables, tanto en las instalaciones sanitarias, en el lavado de vehículos y uso en los jardines.

Simplificando, un sistema de aprovechamiento de aguas pluviales consiste en la captación de agua de lluvia que cae sobre los techos de las edificaciones, el agua es conducida hasta el área de almacenamiento a través de canales o conductores, pasando por equipos de filtrado y descarte de impurezas. Corrigir a partir da linha 20 da página 3 para: "Como la primera porción de agua que pasa por la superficie del techado, posee grande cantidad de suciedad, e incluso se encuentran heces de animales como el caso de aves, roedores, etc. por lo que, es necesario el descarte de las primeras captaciones de aguas (agua de limpieza) a través de dispositivos de desviación (by pass). De acuerdo con Tomaz (2003), dependiendo del material utilizado en el techado, la contaminación puede ser mayor. Por lo que, el agua es almacenada generalmente en un reservorio enterrado (tanque cisterna) y bombeada a un segundo reservorio (punto elevado), del cual son suministradas mediante tuberías instaladas específicas para el agua pluvial y distribuidas para el consumo no potable (May, 2004).

Varios autores como Lade et al. (2013), Mckensie et al. (2013) y Zhang et al. (2013) apoyan al tratamiento de este tipo de aguas antes de ser usadas.

En lo que se refiere a la legislación brasilera relacionada a las aguas pluviales puede verificarse en el Código de Aguas de 1934, y la norma brasilera para el agua de lluvia aprovechamiento en áreas urbanas para fines no potables - NBR 15527/2007 (ABNT, 2007), que trata de los procedimientos y requisitos necesarios al dimensionamiento del sistema de aprovechamiento de las aguas de lluvia para fines no potables. En ella son descritas las condiciones de los componentes de un sistema de aprovechamiento y la calidad requerida para el agua de acuerdo con la utilización prevista. También son dispuestos los métodos de cálculos para el diseño de reservorios.

El objetivo de este estudio fue evaluar la calidad del agua meteórica en la ciudad de Itajubá, ubicado en la parte sur de Minas Gerais, Brasil. Para ello, se realizaron la colecta y análisis de la calidad de las muestras de agua de lluvia después de escurrir sobre la superficie de tres tipos de materiales; amianto, zinc y cerámica. También se realizó una colecta directa para comparar los resultados y verificar los cambios en la calidad del agua.

\section{MATERIAL Y MÉTODOS}

La instalación experimental está ubicada al lado del lago de la Universidad Federal de Itajubá (UNIFEI), es un lugar con bajo flujo de vehículos y se encuentra a una distancia de 100 metros de la avenida principal. 
Los muestreos de las aguas meteóricas fueron realizados en fechas fuera de la temporada de lluvia, para poder evaluar la calidad del agua durante la época de menor disponibilidad de agua de lluvia. Por tanto, durante los meses de marzo a octubre se monitoreó la instalación experimental, especialmente en los días lluviosos; 30/mar (día 1), 31/mar (día 2), 5/abr (día 7), 6/abr (día 8), 24/abr (día 26), 19/may (día 51), 26/may (día 58), 31/may (día 63), 28/set (día 183) y 24/oct (día 209). Así mismo, las diez muestras de agua de lluvia fueron colectadas durante el periodo de investigación, y para caracterizar la influencia del tipo de material de techado en la calidad de agua colectada, fueron montadas tres áreas techadas de 1 $\mathrm{m} 2$ de materiales de amianto, zinc y cerámica, como se muestra en la Figura 1. De la misma forma, fueron instalados canales de PVC (policlorato de vinilo). El montaje experimental fue colocado a cielo abierto y el agua de lluvia fue colectada en recipientes de $2 \mathrm{~L}$.

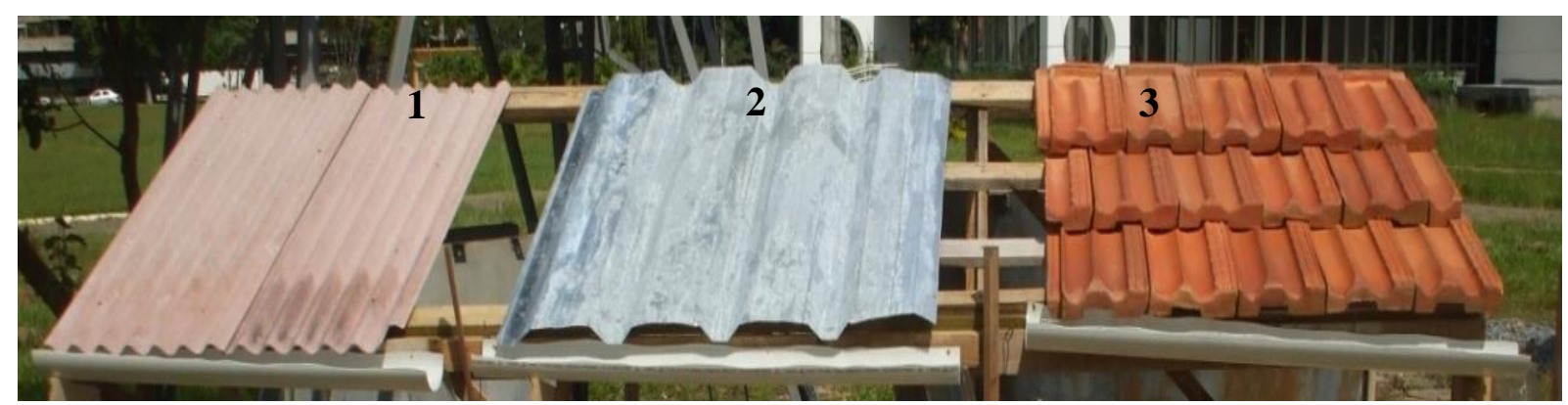

Figura 1. Montaje experimental con superficies de (1) amianto, (2) zinc y (3) cerámica.

Para la colecta directa del agua de lluvia se utilizó una superficie plástica con diámetro de $74 \mathrm{~cm}$ conectado a un recipiente de $10 \mathrm{~L}$.

La limpieza de los recipientes de colecta incluyendo todo el montaje experimental para la colecta directa del agua de lluvia, fue realizada de manera periódica, así mismo en periodos largos de estiaje para evitar la contaminación y garantizar la mayor precisión en los resultados.

Los parámetros controlados fueron elegidos de acuerdo con la relevancia asociada a la utilización de las aguas de lluvia; pH, alcalinidad, dureza, turbidez, coliformes totales y termotolerantes. Las metodologías aplicadas para la determinación analítica de cada variable se encuentran en los Métodos Estándar para el Examen del Agua y Aguas Residuales (APHA, 1998). Los análisis fueron realizados en el Laboratorio de Saneamiento del Instituto de Recursos Naturales en la Universidad Federal de Itajubá.

La caracterización de las muestras fue obtenida a través de las siguientes pruebas: Temperatura, fue obtenida utilizando un termómetro de mercurio; $\mathrm{pH}$, valores obtenidos por técnica potenciométrica; Alcalinidad total, datos obtenidos por la titulación en ácido clorhídrico $(\mathrm{HCl}, 0,02 \mathrm{~mol} / \mathrm{L})$; Dureza, parámetro obtenido por la titulación en solución patrón de EDTA $(\mathrm{N}=1,0169)$; Coliformes totales y termotoleantes, parámetro determinado por la técnica del Colitert; Sólidos totales, suspendidos y volátiles, valores determinados por el método gravimétrico; Sólidos sedimentables, valor obtenido a partir del test del Cono Imhoff.

\section{RESULTADOS Y DISCUSION}

El municipio de Itajubá está localizado en la región sur del estado de Minas Gerais, en Brasil, y según el IBGE Instituto Brasilero de Geografía y Estadística (2014), se estima una población de 94,940 habitantes en el 2013, ocupa un área de $294,835 \mathrm{~km}^{2}$, cuenta aproximadamente con 38,794 vehículos y un parque industrial de 3,631 unidades, presenta un clima tropical de altitud y lluvias intensas concentradas en el verano. Posee un índice pluviométrico medio anual de $1417 \mathrm{~mm}$ y sufre crecidas de ríos e inundaciones, el cual es 
objeto de preocupación y reclamos por parte de la población afectada. Sin embargo, las lluvias en Itajubá no son culpables mas si la ocupación de las áreas de valle, la impermeabilización de las calles y la obstrucción de los ríos, o sea, son interferencias de la propia población en el ambiente natural.

Una de las medidas que pueden contribuir para aumentar el intervalo entre estas inundaciones es el aprovechamiento de las aguas de lluvia que son abundantes en la región del Sur de Minas Gerais y en conjunto, trabajar cada vez más en la sensibilización de la población, para que venga a sentirse responsable por el bien común.

Durante la investigación, se observó que la temperatura oscilaba entre 20 y $26{ }^{\circ} \mathrm{C}$ y fue constante en las muestras colectadas en el mismo día, o sea la temperatura del agua de lluvia no depende del tipo de material de la superficie en el cual escurre el agua colectada, más si de la temperatura del ambiente. Con los datos obtenidos de $\mathrm{pH}$, se graficaron las barras en la Figura 2. Hasta el día 26, no se consideraron los datos de $\mathrm{pH}$ de la muestra directa debido a falla experimental.

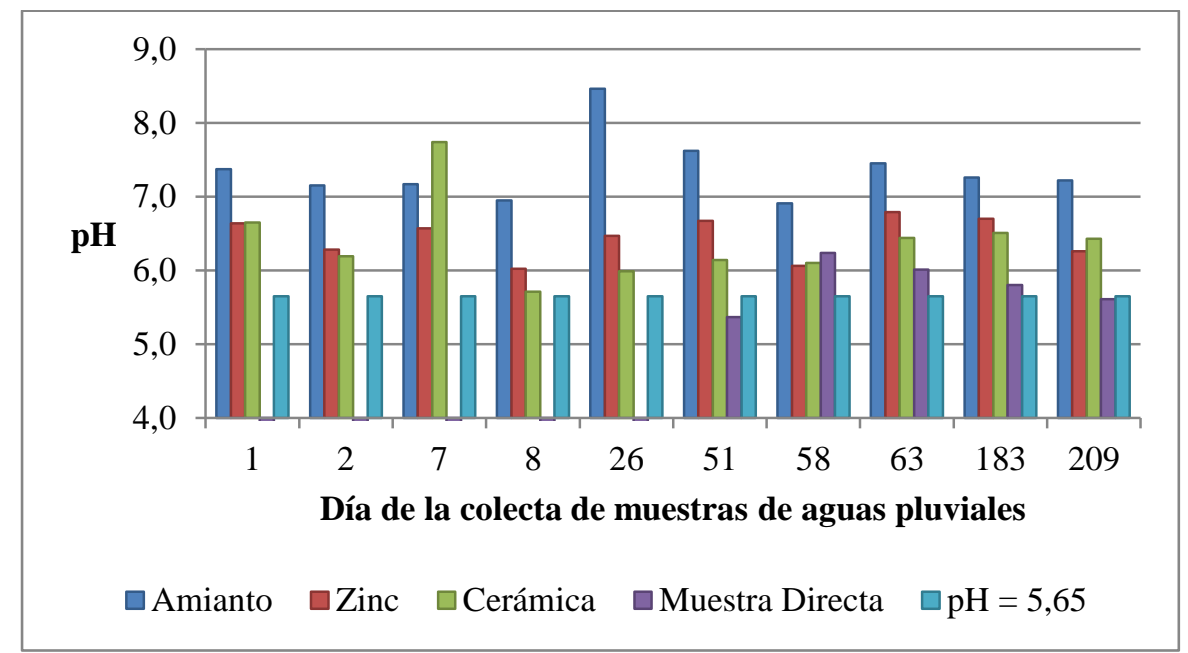

Figura 2. Valor de $\mathrm{pH}$ de las muestras de aguas meteóricas.

Se verifica que el valor medio obtenido para el agua de lluvia colectada directamente fue de $5.81 \pm 0.34$, mientras que las muestras colectadas después de escurrir sobre las superficies de techados presentaron valores mayores de $\mathrm{pH}$ que las anteriores. De acuerdo con la revisión de la literatura realizada, la lluvia ácida es caracterizada por el $\mathrm{pH}$ menos que 5.65, por lo tanto de manera general se puede concluir que en Itajubá no existe problema con relación a las lluvias ácidas. No en tanto, de acuerdo con la norma ABNT NBR 15527/2007 para el aprovechamiento de estas aguas habría la necesidad de ajustar el $\mathrm{pH}$, ayudando a la protección de las instalaciones tubulares.

Las muestras de agua de lluvia que escurren por la superficie de amianto presentaron valores mayores de $\mathrm{pH}$ con una media de $7.36 \pm 0.44$. El $\mathrm{pH}$ obtenido para las muestras que escurrieron por la superficie de zinc, presentaron valores intermedio con una media de $6.45 \pm 0.27$. Y para las muestras que pasaron por la superficie de cerámica los valores de $\mathrm{pH}$ fueron inferiores con una media de $6.39 \pm 0.55$. Hubo un comportamiento inesperado en la colecta de agua de lluvia en la superficie de cerámica, se presentó en el día 3, el cual da un margen para pensar que se trata de un outlier.

Solo fue posible tomar informaciones concluyentes sobre el efecto del $\mathrm{pH}$ para la superficie de amianto, observando que la curva mostrada es significativamente desplazada para el carácter básico en comparación a las demás, con excepción del outlier descrito. De la misma forma se observa que los valores de los datos colectados muestran que el $\mathrm{pH}$ de los 
otros dos tipos de superficie de techado se encuentra relativamente próximo a la muestra colectada al aire libre directo.

El valor medio de alcalinidad obtenido para el agua de lluvia colectada directamente en la atmósfera fue de $13.60 \pm 4.56 \mathrm{mgCaCO}_{3} \mathrm{~L}^{-1}$. Para las muestras que pasaron por la superficie del techo de zinc y de cerámica, la alcalinidad media fue de $14.80 \pm 4.64 \mathrm{mgCaCO}_{3} \mathrm{~L}^{-1}$ y $14.40 \pm 4.30 \mathrm{mgCaCO}_{3} \mathrm{~L}^{-1}$ respectivamente. Mientras que la alcalinidad media del agua de lluvia después de escurrir sobre la superficie del techado de amianto fue de $40.80 \pm 8.80 \mathrm{mgCaCO}_{3} \mathrm{~L}^{-1}$. El comportamiento observado para las muestras que pasaron por la superficie del techado de amianto era esperado, debido a los valores obtenidos de $\mathrm{pH}$ en la colecta del mismo. Entre tanto que los valores de alcalinidad para el resto de las muestras no presentaron variación entre las mismas.

El comportamiento de la dureza de las muestras fue semejante al de la alcalinidad. La dureza media de las muestras colectadas directamente fue de $10.10 \pm 8.96 \mathrm{mgCaCO}_{3} \mathrm{~L}^{-1}$. Para las muestras que pasaron por la superficie del techo de amianto, la dureza media fue de $49.95 \pm 22.94 \mathrm{mgCaCO}_{3} \mathrm{~L}^{-1}$. No en tanto, que la dureza media del agua colectada después de escurrir por la superficie del techado de zinc fue de $13.61 \pm 7.97 \mathrm{mgCaCO}_{3} \mathrm{~L}^{-1}$ y las muestras que escurrieron sobre el techo de cerámica presentaron dureza media de $11.13 \pm 7.07$ $\mathrm{mgCaCO}_{3} \mathrm{~L}^{1}$. De manera general, las aguas de lluvia de Itajubá pueden ser clasificadas como blandas, mientras que las aguas que escurrieron por el techado de amianto pueden ser clasificadas como de dureza blanda a moderada.

El agua de lluvia colectada después de escurrir por los techados de zinc e cerámica no presentaron variaciones significativas con relación a la obtenida directamente en la atmósfera. Entre tanto que las variaciones observadas para las muestras que escurrieron sobre el techado de amianto pueden ser justificadas por la composición química del mismo. El cemento de amianto está compuesto por más de $90 \%$ de cemento y menos de $10 \%$ de fibras de amianto crisotila, cuya fórmula química es $\mathrm{Mg}_{3} \mathrm{SI}_{2} \mathrm{O}_{5}(\mathrm{OH})_{4}$. De acuerdo con Libânio (2005), la dureza indica la concentración de cationes multivalentes en solución en el agua, principalmente de calcio $\left(\mathrm{Ca}^{+}\right)$y magnesio $\left(\mathrm{Mg}^{2+}\right)$. Por tanto, la presencia de iones de magnesio, es uno de los responsables por el aumento de la dureza en el agua, siendo una posible justificación para el comportamiento presentado e incluso pudiendo haber influenciado en los valores de $\mathrm{pH}$ y alcalinidad.

Por medio de la Figura 3, se presenta la turbidez de las muestras analizadas.

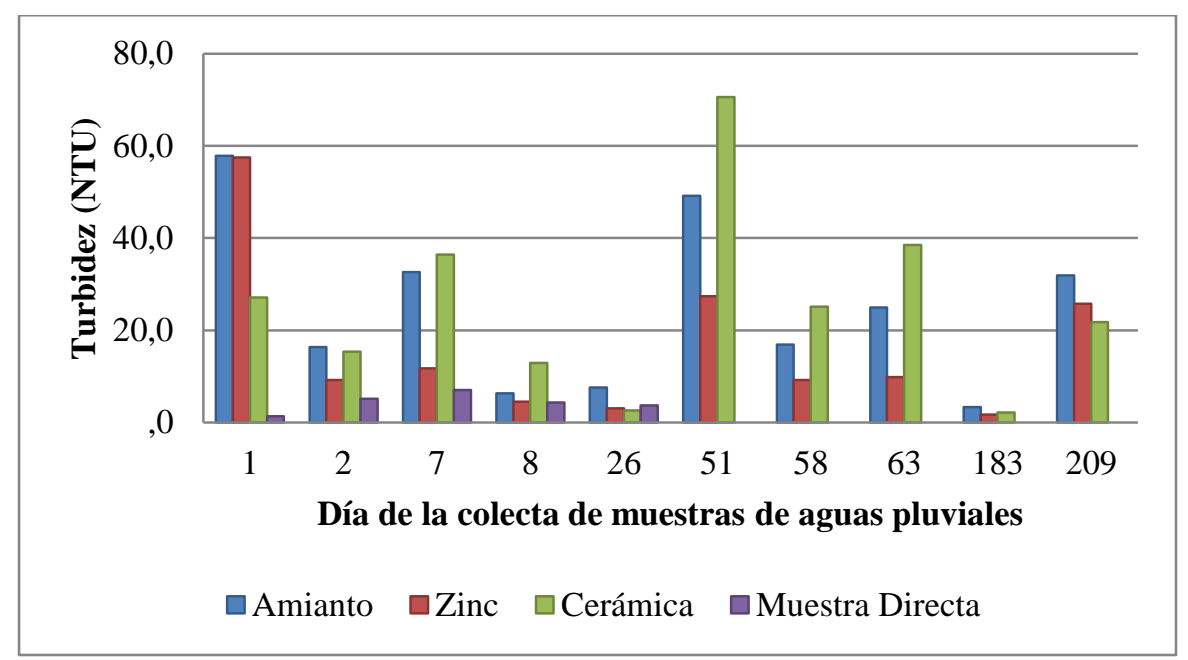

Figura 3. Turbidez de las muestras de aguas meteóricas.

Analizando el comportamiento en la Figura 3, se verifica que las muestras colectadas directamente a la atmósfera, presentaron valores menores de turbidez, con un valor medio de 
$4.33 \pm 2.04$ NTU. Mientras que, para las muestras restantes, es posible observar la variación de la turbidez y se manifiesta aproximadamente en forma homogénea. Se nota que hubo una caída en los valores de turbidez de la colecta 1 para la 2 (del día 1 al 2 del monitoreo) y lo mismo ocurrió en la colecta 3 para el 4 (del día 7 para el día 8 de monitoreo); siendo que en el intervalo sin lluvia entre la colecta 2 y 3 (del día 2 hasta el día 7), hubo un aumento en los valores de turbidez. Este resultado demuestra que para las lluvias en días consecutivos, la primera colecta retira gran parte de las impurezas que toman parte en la turbidez del agua. En los periodos no lluviosos, esas impurezas tienden a acumularse en la superficie del techado.

Los resultados para los sólidos sedimentables solamente se obtuvieron para las muestras colectadas después de 63 días de monitoreo, devido a que el volumen colectado para las demás muestras fue insuficiente para el ensayo del test de Cono de Imhoff. Se verifica que los valores de sólidos sedimentables fueron muy bajos con relación a todas las muestras, no superaron al valor de $1 \mathrm{~mL} \mathrm{~L}^{-1}$. A pesar da la limpieza periódica de los materiales utilizados tratando de minimizar la acumulación de polvo y la obtención de resultados más precisos, los valores encontrados para la muestra colectada directamente pueden tener origen de partículas finas presentes en la atmósfera en cuanto es depositada en los recipientes de la colecta. Es importante resaltar que fueron encontradas cenizas en la muestra del día 183, proveniente de quemadas próximas al área de la colecta de las muestras.

Los contenidos de sólidos totales (ST), sólidos totales fijos (STF) y sólidos totales volátiles (STV) de las muestras son presentados en la Tabla 1.

Tabla 1. Sólidos totales (ST), sólidos totales fijos (STF) y sólidos totales volátiles (STV) en $\mathrm{mg} \mathrm{L}^{-1}$ por día de colecta de las muestras y material de la superficie de colecta.

\begin{tabular}{|c|c|c|c|c|c|c|c|c|c|}
\hline \multirow{3}{*}{$\begin{array}{l}\text { Material de la superficie } \\
\text { de coleta }\end{array}$} & \multicolumn{9}{|c|}{ Día de la coleta de muestras de aguas pluviales } \\
\hline & \multicolumn{3}{|c|}{63} & \multicolumn{3}{|c|}{183} & \multicolumn{3}{|c|}{209} \\
\hline & ST & STF & STV & ST & STF & STV & ST & STF & STV \\
\hline Amianto & 93 & 67 & 26 & 96 & 44 & 52 & 160 & 123 & 37 \\
\hline Zinc & 15 & 12 & 3 & 42 & 0 & 42 & 122 & 89 & 33 \\
\hline Cerámica & 48 & 48 & 0 & 68 & 12 & 56 & 93 & 55 & 38 \\
\hline Muestra directa al aire libre & 28 & 16 & 12 & 38 & 7 & 31 & 55 & 22 & 33 \\
\hline
\end{tabular}

Debido a la mayor porosidad de las superficies de amianto y cerámica, se esperaba que las muestras colectadas a partir de ellas poseyesen mayor concentración de sólidos. Analizando la Tabla 1, se observa que las muestras colectadas después que escurrieron sobre la superficie de amianto y cerámica en los días 63 e 183 presentaron mayor concentración de sólidos que el de las muestras que escurrieron por la superficie de zinc. Mientras que, para las muestras colectadas en el día 209, la muestra obtenida con la cobertura de zinc presentó alta concentración de sólidos. Por lo tanto, no es posible describir informaciones concluyentes, siendo necesario considerar mayor cantidad de muestras para el mejor análisis de los resultados.

Los sólidos también pueden ser determinados indirectamente por la conductividad eléctrica y vice-versa, para ello se aplica un factor de conversión. En este estudio, el factor varió de 0.98 a 9.13. De modo que, la conductividad eléctrica CE puede ser aproximada por la Ecuación 1:

$$
\mathrm{CE}\left(\mu \mathrm{S} \mathrm{cm}^{-1}\right)=\mathrm{ST}\left(\mathrm{mg} \mathrm{L}^{-1}\right) / 3.18
$$

Siendo los siguientes valores de CE para cada superficie evaluada: 
- Muestra directa: 19.0 a $37.4 \mu \mathrm{S} \mathrm{cm}^{-1}$, en media $27.4 \mu \mathrm{S} \mathrm{cm}^{-1}$,

- Amianto: 63.2 a $108.8 \mu \mathrm{S} \mathrm{cm}^{-1}$, en media $79.1 \mu \mathrm{S} \mathrm{cm}^{-1}$,

- Zinc: 10.2 a $83.0 \mu \mathrm{S} \mathrm{cm}^{-1}$, en media $40.6 \mu \mathrm{S} \mathrm{cm}^{-1}$,

- Cerámica: 32.6 a $63.2 \mu \mathrm{S} \mathrm{cm}{ }^{-1}$, en media $47.4 \mu \mathrm{S} \mathrm{cm}^{-1}$

Según CETESB (2013), en general, los valores de CE mayores que $100 \mu \mathrm{S} \mathrm{cm}^{-1}$ indican que la calidad del agua fue alterada. Mientras que para fines de irrigación, Cordeiro (2001) clasifica que las aguas con conductividad menor a $250 \mu \mathrm{S} \mathrm{cm}^{-1}$ son aguas de salinidad baja. Por lo tanto, se puede constatar que las aguas meteóricas de Itajubá aparentemente no presentan ese problema.

Los resultados determinados en los análisis de coliformes totales y termotolerantes están descritos en las Tabla 2. Vale resaltar que algunas muestras no contaban con volumen suficiente para a la realización del análisis bacteriológica.

Tabla 2. Coliformes totales y termotolerantes $\left(\mathrm{NMP} \mathrm{mL}^{-1}\right)$ por día de colecta de muestras y material de la superficie de coleta.

\begin{tabular}{|c|c|c|c|c|c|c|c|}
\hline \multirow{2}{*}{$\begin{array}{l}\text { Material de la } \\
\text { superficie de coleta }\end{array}$} & \multicolumn{7}{|c|}{ Día de la colecta de muestras de las aguas pluviales } \\
\hline & 1 & 2 & 7 & 26 & 51 & 63 & 183 \\
\hline \multicolumn{8}{|l|}{ Coliformes totales } \\
\hline Amianto & $>24,196$ & $>24,196$ & $>24,196$ & 0,771 & $>24,196$ & 0,233 & 1,981 \\
\hline Zinc & 0,031 & $>24,196$ & $>24,196$ & 0 & 0 & 0 & 0 \\
\hline Cerámica & $>24,196$ & $>24,196$ & $>24,196$ & 0,090 & $>24,196$ & 1,789 & 0,767 \\
\hline $\begin{array}{l}\text { Muestra directa al aire } \\
\text { libre }\end{array}$ & - & - & - & - & 0,448 & 0,441 & 0 \\
\hline \multicolumn{8}{|c|}{ Coliformes termotolerantes } \\
\hline Amianto & 0,207 & $>24,196$ & $>24,196$ & 0 & $>24,196$ & 0,010 & 0,087 \\
\hline Zinc & 0 & $>24,196$ & $>24,196$ & 0 & 0 & 0 & 0 \\
\hline Cerámica & 0,124 & $>24,196$ & $>24,196$ & 0 & $>24,196$ & 0 & 0,010 \\
\hline $\begin{array}{l}\text { Muestra directa al aire } \\
\text { libre }\end{array}$ & - & - & - & - & 0,099 & 0,031 & 0 \\
\hline
\end{tabular}

A partir del análisis de los datos obtenidos, se verifica que los valores de coliformes totales y termotolerantes presentaron variaciones, indicando desde ausencia como el valor máximo encontrado en el test de Colilert@.

Ese resultado demuestra que existe contaminación por heces de animales de sangre caliente como aves y gatos, el hecho fue comprobado pues se encontraron heces de animales sobre las superficies de los techos durante el periodo de colecta de agua de lluvia. También, es interesante notar, que la muestra escurrida por el techo de zinc presentó ausencia de microrganismos en tres muestras. Se considera que el techo de zinc, por ser metálica, refleja la radiación solar que incide sobre ella misma, por tanto, se comprueba el poder de inactivación de microrganismos. Y las demás superficies de techos empleados, poseen mayor porosidad siendo y por ello es favorable para la presencia de microrganismos potencialmente patogénicos. 
A pesar de deducir que la muestra colectada directamente fuese exenta de coliformes totales y termotolerantes, los resultados obtenidos demuestran que existió contaminación en el agua de lluvia colectada, posiblemente se debe a la contaminación en la superficie de la colecta. Por otra parte, un análisis más preciso requiere mayor cantidad de muestras. No en tanto, habrá indicación de una desinfección necesaria del agua colectada para su posterior utilización.

Comparando los datos de turbidez y coliformes es posible observar que las muestras que corresponden a los mayores valores de coliformes, también, presentaron turbidez elevada, indicando la importancia de ser tratados las aguas de lluvia antes de su almacenamiento y aprovechamiento. Aún se verifica, que las muestras después de escurrir por las superficies de cerámica y amianto presentaron valores superiores de turbidez en comparación con las muestras que escurrieron por el techo de zinc. Esto puede ser explicado por la diferencia de porosidad de los materiales, pues los techos de cerámica y amianto poseen mayor porosidad que las tejas de zinc.

\section{CONCLUSION}

A partir de los resultados analizados se concluye que existe influencia del material de techo en la calidad del agua de lluvia que escurre sobre el mismo. Dependiendo de la composición química del material, puede haber reacciones con el agua que escurre en su superficie, modificando parámetros como el $\mathrm{pH}$, alcalinidad y dureza del agua colectada. La porosidad del material, también es un factor que puede crear condiciones favorables a la proliferación de microrganismos o retener mayor cantidad de partículas, proveyendo turbidez al agua o la mayor cantidad de sólidos.

También, se verifica que existe influencia del ambiente que se encuentra el material del techo, pues fueron detectados ramas de árbol, cenizas, heces y plumas de animales en algunas muestras, favoreciendo la contaminación del agua de lluvia. Por lo que, es posible confirmar que el uso de las aguas pluviales deben ser prioritariamente para fines no potables, debido a la grande dificultad de ser controlado estos factores en un sistema de aprovechamiento del agua de lluvia.

Además, se puede concluir que las aguas pluviales antes de ser usadas deben ser tratadas para no ofrecer riesgos a la salud de las personas que entraren en contacto con esta agua y para preservar la integridad de las instalaciones de tuberías, equipos y reservorios. Comparando los resultados obtenidos con lo que establece la norma ABNT NBR 15527/2007, se recomienda que el tratamiento de estas aguas deben prever la remoción de sólidos gruesos y finos, el ajuste del pH y la desinfección, pues el uso de agua sanitariamente segura previene la aparición de varias enfermedades como leptospirosis, disentería, cólera y otras.

El uso de las aguas de lluvia aún no está sujeto a cobranzas, al contrario de otros tipos de aguas. Además, de ser accesible a todos, independiente de la condición social o económica, puede contribuir por dos cuestiones principales relacionadas al agua: (i) disminución de la demanda de agua de abastecimiento, por el almacenamiento del agua de lluvia en cisternas o reservorios, preservando los manantiales superficiales y subterráneos, y (ii) disminución de los picos de volumen de agua en los lechos de los ríos, mediante la retención temporal de los volúmenes de agua de lluvia, captados en las superficies impermeables de los terrenos y almacenados en reservorios, disminuyendo con esto la magnitud de las inundaciones urbanas. Por lo tanto, los habitantes de Itajubá pueden ser beneficiados con el potencial de las aguas meteóricas existentes en este lugar, que poseen bajo grado de contaminación, a fin de maximizar su uso en favor del desarrollo sustentable de la ciudad. 


\section{REFERENCIAS}

AMERICAN PUBLIC HEALTH ASSOCIATION - APHA. Standard methods of the examination of water and wastewater. 20. ed. Washington, 1998. 1 CD-Rom.

ASSOCIAÇÃO BRASILEIRA DE NORMAS TÉCNICAS. NBR 15527 / 2007: água de chuva - aproveitamento de coberturas em áreas urbanas para fins não potáveis Requisitos. Rio de Janeiro, 2007. 8p.

AZEVEDO NETTO, J. M. Aproveitamento de águas de chuva para abastecimento. Revista BIO, Rio de Janeiro, ano 3, n. 2, p. 44-48, 1991.

CAMPOS, M. A. S.; HERNANDES, A. T.; AMORIN, S. V. Análise de custo da implantação de um sistema de aproveitamento de água pluvial para uma residência unifamiliar na cidade de Ribeirão Preto. In: SIMPÓSIO BRASILEIRO DE CAPTAÇÃO E MANEJO DE ÁGUA DE CHUVA, 4., 09 a 11 julho, 2003, Juazeiro. Uma proposta sustentável para o combate à fome e para a melhoria da qualidade de vida. Juazeiro: ABCMAC, 2003. .

COMPANHIA AMBIENTAL DO ESTADO DE SÃO PAULO - CETESB. Relatório de qualidade das águas superficiais no estado de São Paulo 2012. Apêndice C Significado ambiental e sanitário das variáveis de qualidade. São Paulo: 2013. 36p.(Relatórios).

CORDEIRO, G. G. Qualidade de água para fins de irrigação: conceitos básicos e práticos. Petrolina: Embrapa Semi-Árido, 2001. 34p. (Documentos, 167).

EATON, J. S.; LIKENS, G. E.; BORMANN. H. Throughfall and stemflow chemistry in a Northern Hardwood Forest. The Journal of Ecology, London, v. 61, n. 2, p. 495-508, jul. 1973. http://www.jstor.org/stable/2259041

GNADLING, J. Captação de água da chuva para produção de alimentos: a experiência do estado de Gansu no norte da China. In: SIMPÓSIO SOBRE CAPTAÇÃO DE ÁGUA DA CHUVA, 3., 21-23 novembro 2001, Campina Grande. Anales... Campina Grande: ABCMAC, 2001. 14p.

INSTITUTO BRASILEIRO DE GEOGRAFIA E ESTATÍSTICA - IBGE. Informações estatísticas de Itajubá - MG. Disponible en: 〈http://cod.ibge.gov.br/236VO〉. Acceso en: 19 mayo 2014.

JAQUES, R. C. Qualidade da água de chuva no município de Florianópolis e sua potencialidade para aproveitamento em edificações. 2005. 102f. Dissertação (Mestrado em Engenharia Ambiental) - Departamento de Engenharia Sanitária e Ambiental, Universidade Federal de Santa Catarina - UFSC, Florianópolis, 2005.

KUS, B.; KANDASAMY, J.; VIGNESWARAN, S.; SHON, H.; MOODY, G. Gravity driven membrane filtration system to improve the water quality in rainwater tanks. Water Science \& Technology: Water Supply, United Kingdom, v. 13, n. 2, p. 479-485, 2013. http://hdl.handle.net/10453/23757

LADE, O.; OLOKE, D.; CHINYIO, E.; FULLEN, M. Potential for potable water savings by using rainwater: a case study of Ibadan, Nigeria. International Journal of Advancements in Research \& Technology, v. 2, n. 4, p. 117-131, abr. 2013. 
LEE, M. F.; NAZARUDEEN, H. Collection of urban stormwater for potable water supply in Singapore. Water Quality International, Singapore, p. 36-40, May/June 1996.

LIBÂNIO, M. Fundamentos de qualidade e tratamento de água. Campinas, Átomo, 2005. $444 \mathrm{p}$.

LIBERAL, G. S.; PORTO, E. R. A situação atual de cisternas rurais construídas por programas governamentais. In: SIMPÓSIO SOBRE CAPTAÇÃO DE ÁGUA DE CHUVA NO SEMI-ÁRIDO BRASILEIRO, 2., Petrolina, Anales... Petrolina: ABCMAC, 1999. 3p.

MACKENZIE, E. R.; JENKINS, M. W.; TIWARI, S. K.; DARBY, J.; SAENYI, W.; GICHABA, C. M. In-home performance and variability of biosand filters treating turbid surface and rain water in rural Kenya. Journal of Water, Sanitation and Hygiene for Development, North Carolina, v. 3, n. 2, p. 189-198, 2013. http://dx.doi.org/10.2166/ washdev.2013.050

MAY, S. Estudo da viabilidade do aproveitamento de água de chuva para consumo não potável em edificações. 2004. 189f. Dissertação (Mestrado em Engenharia da Construção Civil) - Escola Politécnica, Universidade de São Paulo - USP, São Paulo, 2004.

RAMOS, A.A. O saneamento em dois tempos Desterro e Florianópolis. Florianópolis: CASAN, 1983. 57p.

SCHMIDT, M. Captação de água da chuva na Alemanha: novos conceitos de substituição de água potável, controle de enchentes e melhora da qualidade da água superficial. In: SIMPÓSIO SOBRE CAPTAÇÃO DE ÁGUA DA CHUVA, 3., 21-23 novembro, 2001, Campina Grande. Anales... Campina Grande: ABCMAC, 2001.

THOMAS, P. R.; GREENE, G. R. Rainwater quality from different roof catchments. Water Science \& Technology, London, v. 28, n. 3-5, p. 291-299, 1993.

TOMAZ, P. Aproveitamento de água de chuva para áreas urbanas e fins não potáveis. 2. ed. São Paulo: Navegar, 2003. 180p.

ZHANG, X. L.; CHEN, J. J.; HU, L. A type of rainwater ecological treatment technology: Rainwater Biofiltration System. Applied Mechanics and Materials, Millersville, v. 295-298, p. 1502-1507, 2013. http://dx.doi.org/10.4028/www.scientific.net/AMM.295298.1502 